\title{
Ultra-Wideband Pulses Generation by means of Incoherent Optical Processing compatible with Radio- over-Fibre Systems
}

\author{
Mario Bolea
}

\author{
Manuel Rius, José Mora, José Capmany
}

\begin{abstract}
A technique for UWB pulses generation based on incoherent optical signal processing through a dispersive element is proposed and experimentally demonstrated. The generated waveform is given by the optical signal power distribution. Therefore, waveform reconfiguratiblity is achieved by the proper control of the incoherent optical signal power distribution. UWB pulses generation have been experimentally demonstrated by means of the synthesis of monocycle and doublet waveforms using an optical channel selector to control the optical signal power distribution. Moreover, for wireless communications, the complete control on the waveform permits to fulfil the FCC spectral requirements and thus an increasing of the UWB radio signals coverage area.
\end{abstract}

Keywords-UWB communications; monocycle; doublet; optical signal power spectral density; FCC mask

\section{INTRODUCTION}

In last times, the interest on Ultra-Wideband (UWB) technology has considerably increased due to the wide variety of commercial applications such as wireless communications, sensor networks and radar imaging. Particularly, UWB for broadband wireless communications is a topic of interest due to characteristics such as its ability to overlay existing band occupants without interfering, potentially high data rates, its high temporal resolution and the capability to penetrate through the obstacles. Nevertheless, the low power density regulated by the Federal Communications Commission (FCC) limits the radio communication distances, typically extending less than tens of meters [1]. In this point, the use of optical fibre for UWB signals distributions emerges as a promising solution to increase the coverage area what is known as UWB-over-fibre. In addition to the distribution of UWB over optical fibre, it is also desirable that UWB signals can be generated and encoded directly in the optical domain without the need of extra opticalelectrical and electrical-optical conversions, to fully exploit the advantages provided by optics [2].

A great number of approaches have been reported to generate UWB waveforms in the last decade. We can found schemes to generate classical UWB pulses, i.e. monocycles and doublets, based on phase-modulation-to-intensity-modulation (PM-TM) conversion by using an optical frequency discriminator implemented by optical filters [3]. Microwave photonic filters have been also proposed for UWB signals generation implementing negative taps based on the nonlinear amplification processing in a SOA [4] and the combination of the effects of cross-phase modulation in a SOA-MZI [5]. We can also find schemes based on optical spectral shaping [6] and dispersion-induced frequency-to-time mapping [7].

One of the key aspects to take into account for the UWB waveform generation is the adaptation to the FCC mask. In this way, if a transmission system implements waveforms which are adapted to the FCC mask, higher power spectral efficiencies can be achieved since no-extra attenuation must be introduced. Therefore, the coverage distance for wireless communications can be increased.

In order to overcome this lack of FCC fitting, we propose a technique for generating UWB pulses based on the processing of incoherent signals by means of dispersive elements. Positive and negative pulses are easily achieved through the corresponding shaping of the incoherent optical signal. The amplitude and polarity of these optical pulses can be controlled independently. Moreover, the propagation through a dispersive element introduces the corresponding difference time delay between pulses in order to generate different UWB waveforms. Particularly, monocycle and doublet UWB pulses generation have been demonstrated. Furthermore, the control over the optical source power profile permits to overcome limitations around GPS band (0.96-1.61 GHz) of the FCC mask. This fact permits avoid

\section{SYSTEM PERFORMANCE}

The block diagram shown in Fig. 1 corresponds to the proposed UWB pulses generator. First of all, the optical source is composed by a Broadband Source (BBS) and an Optical Channel Selector (OCS). The BBS has a total optical bandwidth of $80 \mathrm{~nm}$ and the OCS is centered at $1546.92 \mathrm{~nm}$ with 48 channels of $0.8 \mathrm{~nm}$ width. The attenuation of each channel can be controlled independently up to a maximum of $20 \mathrm{~dB}$ in order to achieve different optical power distributions. Next, the shaped optical signal is modulated by a RF pulse using a Mach-Zehnder Modulator (MZM). This RF pulse $\left(\mathrm{p}_{\mathrm{RF}}(\mathrm{t})\right)$ is obtained from a $\mathrm{RF}$ pulse generator which is 


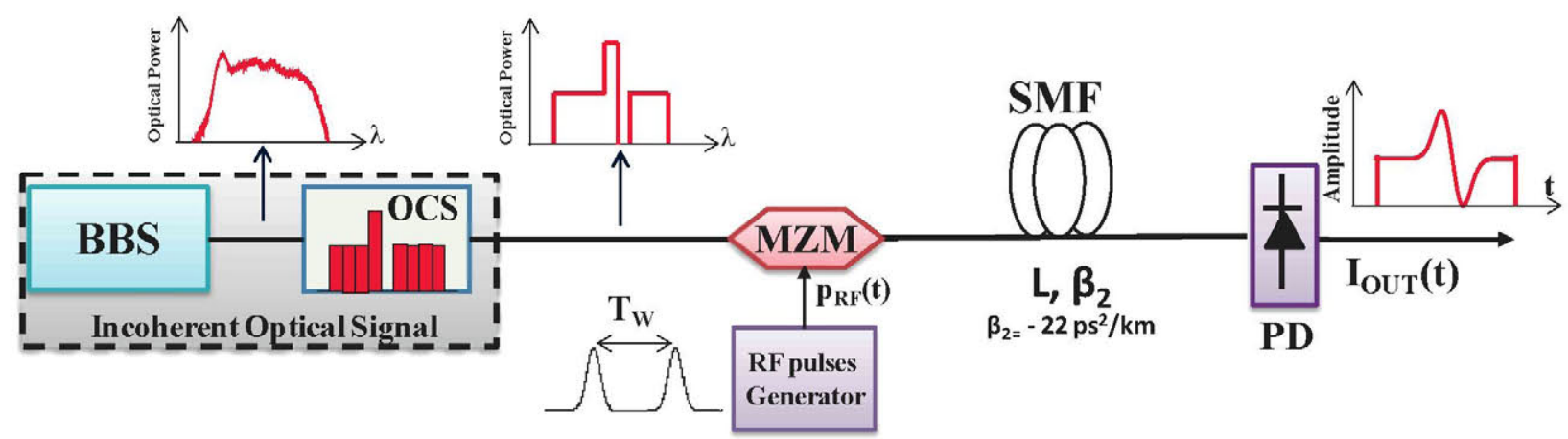

Fig. 2. Scheme of the UWB pulses generator

configured at a bit rate of $12.5 \mathrm{~Gb} / \mathrm{s}$ with a pattern of one "1" and sixty-three " 0 ". Therefore, the repetition rate is close to 195 $\mathrm{MHz}\left(1 / \mathrm{T}_{\mathrm{W}}\right)$ and the pulse width is $80 \mathrm{ps}$. The modulated optical signal is propagated through a standard single mode fibre link (SMF) with a length of $5.43 \mathrm{~km}$ and a first order dispersion parameter $\beta_{2}=-22 \mathrm{ps}^{2} / \mathrm{km}$. Finally, the resulting generated waveform ( $\mathrm{I}_{\text {OUT }}(\mathrm{t})$ ) is obtained after the photodetection $(\mathrm{PD})$ process.

In order to show the performance of the system, positive and negative pulses have been generated. By means of the OCS, the optical source spectrum is shaped to a power spectral density shown in Fig. 2(a). In this case, all OCS channels are attenuated in order to obtain a uniform power distribution at the half of the maximum optical power available. However, the channel at $1546.92 \mathrm{~nm}$ has been maintained with the maximum power. The corresponding signal generated is plotted in Fig. 2(c). As can be observed, a positive pulse is obtained on a uniform pedestal. On the other hand, the channel at 1547.72 $\mathrm{nm}$ is totally attenuated as shown Fig. 2(b). The corresponding generated pulse is depicted in Fig. 2(d). In this case, a pulse with negative polarity has been achieved. Therefore, electrical pulses with positive and negative polarity can be generated with the control of the OCS attenuation of each channel.
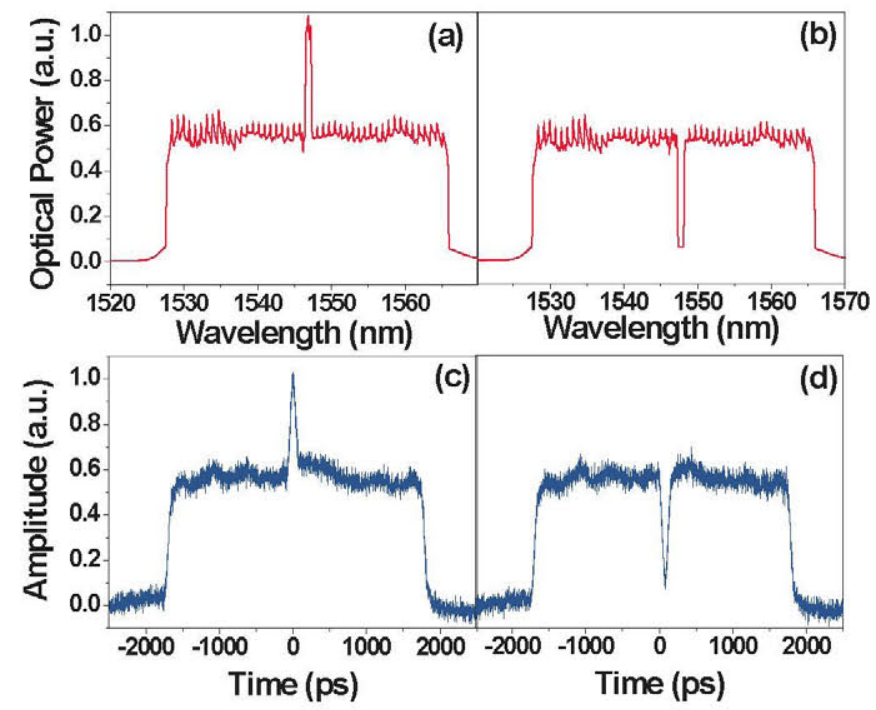

Fig. 2. Optical source power profile for a (a) positive and (b) negative pulse. Generated waveform for (c) positive and (d) negative pulse.

\section{UWB PULSES GENERATION}

In this section, we combine different positive and negative pulses to generate UWB signals as it is shown in Fig. 3.
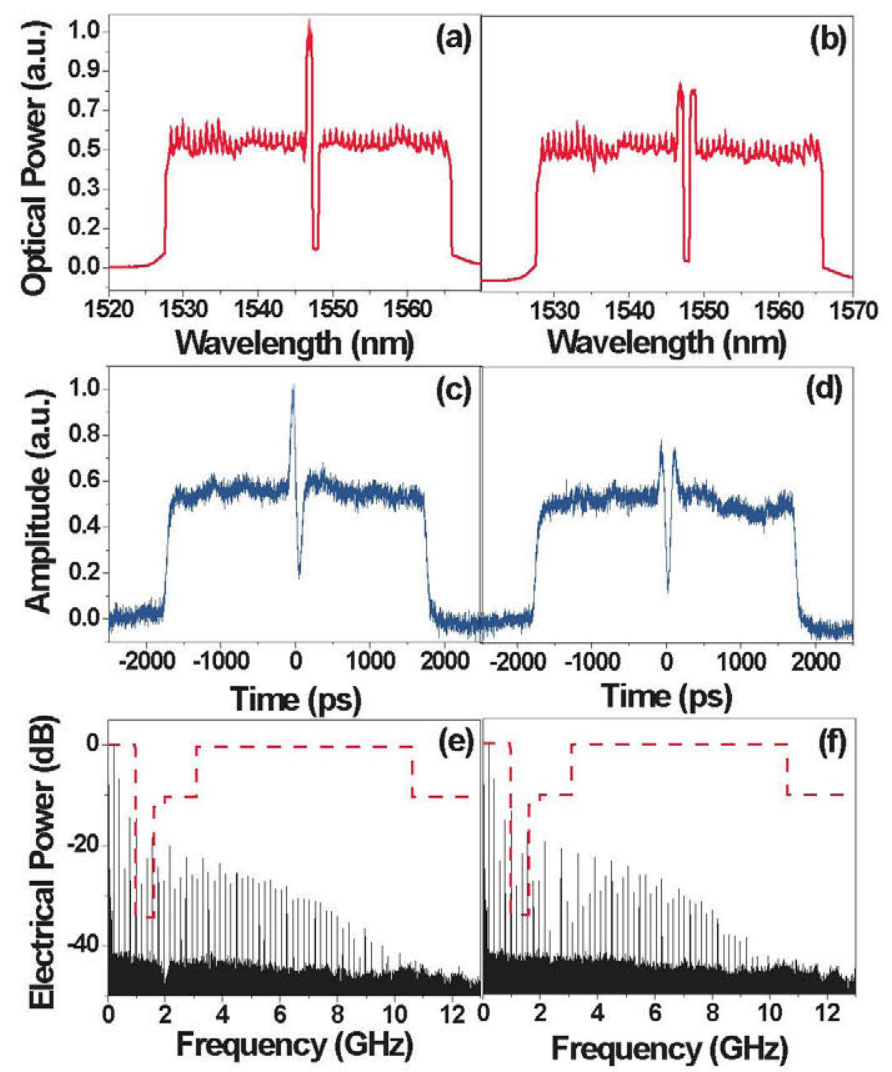

Fig. 3. Experimental results with a uniform pedestal of the optical source power spectral density, waveform and corresponding electrical spectrum for monocycle (a), (c), (e) and doublet (b), (d), (f), respectively.

First of all, a monocycle pulse is generated. The optical source is adjusted according to the power profile distribution shown in Fig. 3(a) where one channel has the maximum power and the other one is totally blocked. The waveform generated is plotted in Fig. 3(c) which corresponds to a monocycle pulse since both positive and negative lobes have the same amplitude. Therefore, the electrical pulse generated is given by the optical source power profile. On the other hand, a doublet has been obtained by means of using three channels. For this case, the power of the two channels related to positive pulses is 
adjusted to be the half of a channel blocked given a power distribution as shown in Fig. 3(b). The generated waveform (see Fig. 3(d)) corresponds to a doublet pulse. In Figs. 3(e) and $3(\mathrm{f})$, the electrical spectra of the monocycle and doublet are plotted. We have included the FCC mask in dashed line. As can be observed, for both waveforms, the FCC spectral requirements are not fulfilled. In this way, the power transmitted should be reduced leading to a limitation in the coverage area of the UWB system.

The main restriction of the FCC spectral requirements is around de GPS band (0.96-1.61 GHz) since this service operates with very low power rate. The generated waveforms have a wide baseband component due to the pedestal in which they are located. This baseband component is clearly interfering with GPS band and affecting to the system spectral efficiency.

The flexibility of the proposed system permits to control the pedestal in order to reduce the baseband component contribution. In order to show this fact, we obtain the results shown in Fig. 4.
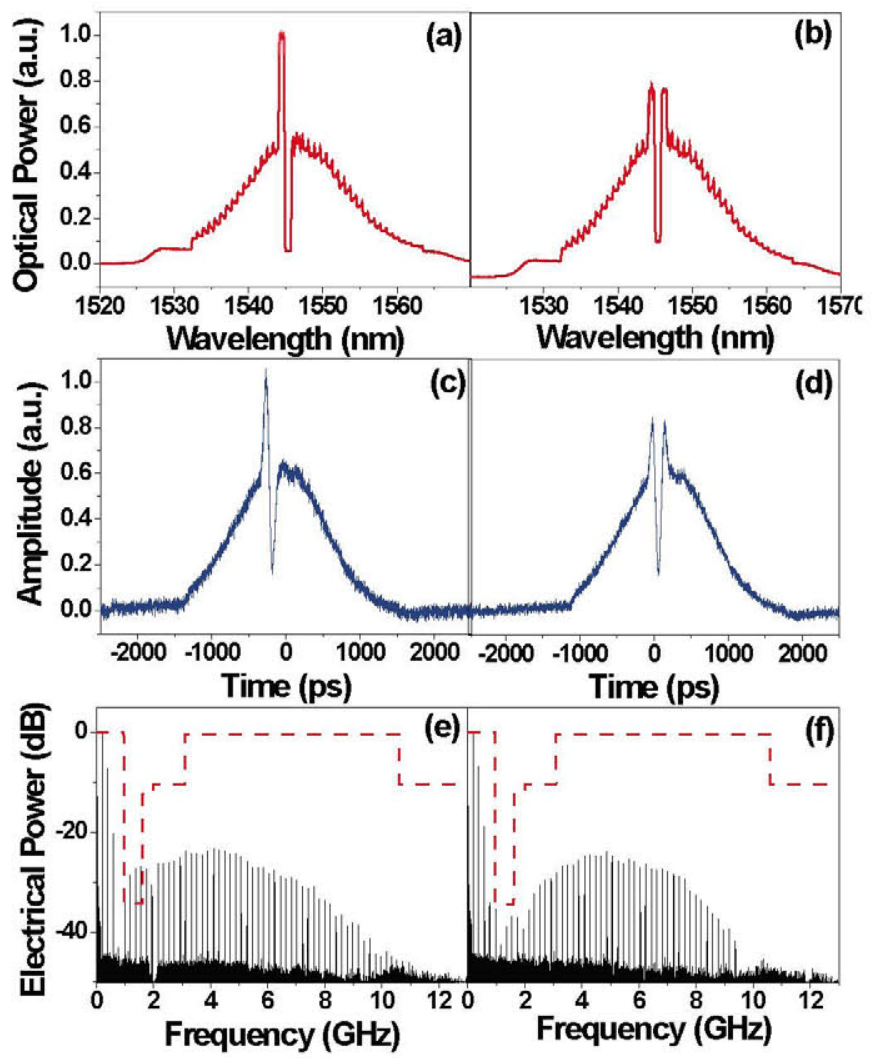

Fig. 4. Experimental results with a gaussian pedestal of the optical source power spectral density, waveform and corresponding electrical spectrum for monocycle (a), (c), (e) and doublet (b), (d), (f), respectively.

In the following, the OCS is reconfigured to adjust the optical source power distribution to a Gaussian profile with 14 nm optical bandwidth. For generating the UWB pulses, we have selected the same configuration of the central channels than Fig. 3. As a result, we obtain the optical signal power distributions shown in Figs. 4(a) and 4(b) for the monocycle and doublet, respectively. Now, the pedestal corresponds to Gaussian shape. Figure 4(e) represents the electrical spectrum measured for the monocycle. Again, the FCC spectral requirements are not fulfilled. However, the power reduction that should be applied in this case is considerably lower than Fig. 3(e). The generated doublet spectrum is shown in Fig. 4(f). As can be observed, in this case, the FCC mask is satisfied avoiding the power reduction. Therefore, the optical source power profile control permits to improve the spectral efficiency and, consequently, the coverage distance of the generated UWB waveforms.

\section{CONCLUSION}

In conclusion, we have proposed and experimentally demonstrated a technique for UWB pulses generation based on the processing of an incoherent optical signal using a dispersive element. The use of incoherent optical sources permits to increase the flexibility in order to reconfigure the generated waveform which is given by the optical signal power profile. The feasibility of the system has been experimentally demonstrated by means of the generation of monocycle and doublet pulses. More importantly, we have proved the capability of the proposed system to improve the spectral efficiency of the waveform generated according to the FCC spectral requirements. Therefore, for wireless communications, this fact permits to avoid the need of reducing the signal power to satisfy the FCC mask thus improving the coverage distance of the UWB radio signals transmitted.

\section{ACKNOWLEDGMENT}

The authors wish to acknowledge the financial support by the National Project TEC2014-60378 funded by the Spanish Ministerio de Economia y Competitividad and the Research Excellency Award Program GVA PROMETEO 2013/012.

\section{REFERENCES}

[1] G. R. Aiello and G. D. Rogerson, "Ultra-wideband wireless systems," IEEE Microw. Mag., vol. 4, pp. 36-47, June 2003.

[2] J. Yao, "Photonics for Ultrawideband Communications," IEEE Microw. Mag., vol. 10, pp. 66-74, June 2009.

[3] V. Moreno, M. Rius, J. Mora, M. A. Muriel and J. Capmany, "Scalable UWB photonic generator based on the combination of doublet pulses," Opt. Exp., vol. 22, pp. 15346-15351, June 2014.

[4] Y. Yu, J. Dong, X. Li, X. Zhang, "UWB monocycle generation and Biphase modulation based on Mach-Zehnder Modulator and semiconductor optical amplifier", IEEE Photon. J., vol. 4, pp. 327-339, April 2012.

[5] V. Moreno, M. Rius, J. Mora, M. A. Muriel and J. Capmany, "Integrable high order UWB pulse photonic generator based on cross-phase in a SOA-MZI," Opt. Exp., vol. 21, pp. 22911-22917, September 2013.

[6] M. Abtahi, M. Mirshafiei, J. Magne, L. A. Rush and S. LaRochelle, "Ultra-Wideband waveform genertor based on optical pulse-shaping and FBG tuning," IEEE Photon. Techn. Lett., vol. 20, pp. 135-137, January 2008.

[7] C. Wang and J. Yao, "All-fiber ultrawideband pulse generation based on spectral shaping and dispersion-induced frequency-to-time-mapping conversion," IEEE Photon. Techn. Lett., vol. 19, pp. 137-139, February 2007. 